Supporting information:

\title{
Synergistic Effect of Singly Charged Oxygen Vacancies and Ligand-Field for Regulating Transport Properties of Resistive Switching Memories
}

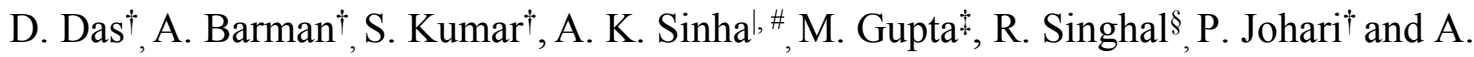

$$
\text { Kanjilal }{ }^{\dagger *}
$$

*Corresponding author: aloke.kanjilal@,snu.edu.in, priya.johari@,snu.edu.in

†Department of Physics, School of Natural Sciences, Shiv Nadar University, NH-91, Dadri, Gautam Buddha Nagar, Uttar Pradesh 201 314, India

ISynchrotron Utilization Section, Raja Ramanna Center for Advanced Technology (RRCAT), Indore, Madhya Pradesh 452013, India

\# Homi Bhabha National Institute, Anishaktinagar, Mumbai-94, India

¥UGC-DAE Consortium for Scientific Research, Khandwa Road, Indore, Madhya Pradesh452001, India

$\S$ Department of physics, Malaviya National Institute of Technology Jaipur, JLN Marg, Malaviya Nagar,Jaipur-302017,India 


\section{S1. X-ray absorption near edge structure (XANES)}

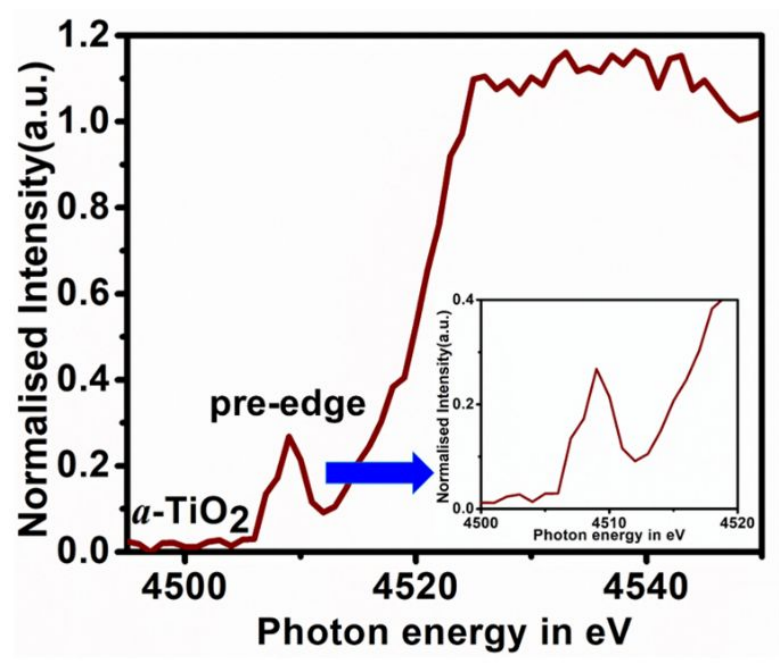

Figure S1. X-ray absorption near edge structure (XANES) spectra at the Ti- $K$ edge, inset is showing the pre-edge feature.

XANES measurements were carried out in fluorescence mode at ambient pressure and room temperature at Indus-2, BL-12 RRCAT India. The details of the experimental setup and the beamline can be found elsewhere. ${ }^{1-2}$ At first, data of the standard anatase- $\mathrm{TiO}_{2}$ powder was recorded. Then, the position of the Ti- $K$ edge was obtained from the standard XAS database of Hephaestus. ${ }^{3}$ Further, energy correction was performed for $a-\mathrm{TiO}_{2}$ samples to get the correct edge position. Finally, spectra were normalised to photon intensity and presented. 
S2. Evolution of $a-\mathrm{TiO}_{2}$ (at various temperatures) structure through Molecular dynamics simulation

a

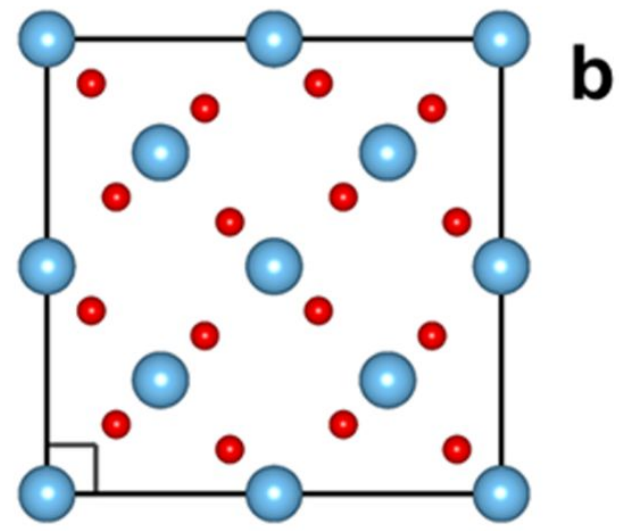

Starting structure $\left(\mathrm{R}-\mathrm{TiO}_{2}\right)$

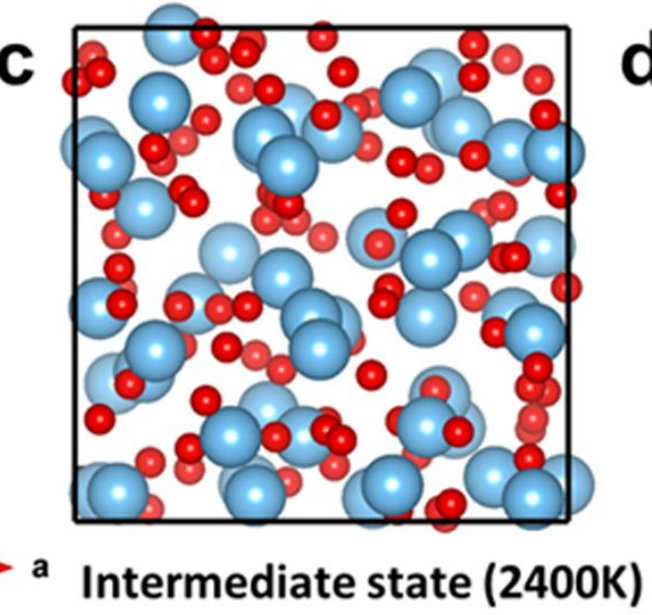

b

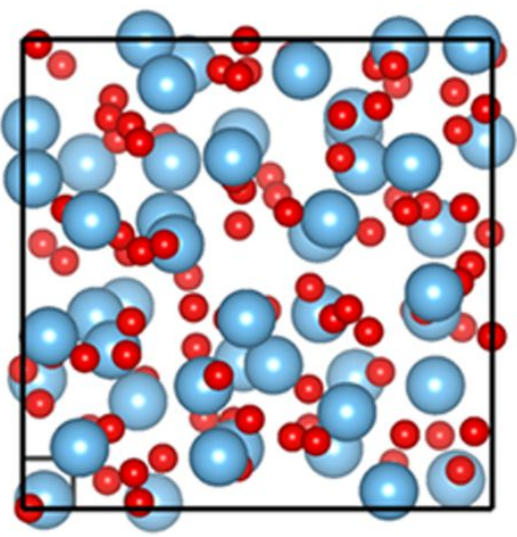

Melted state (4800K)

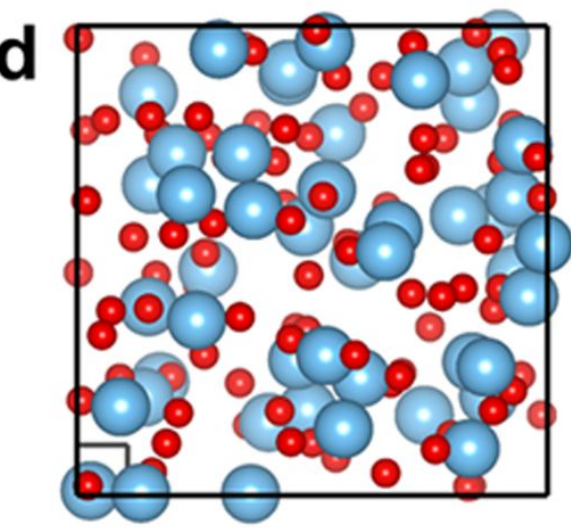

Final state (300K)

Figure S2. Evolution of $a-\mathrm{TiO}_{2}$ structure at various temperatures (a) starting structure $\left(R-\mathrm{TiO}_{2}\right)$ (b) $4800 \mathrm{~K}$ (c) $2400 \mathrm{~K}$ (d) $300 \mathrm{~K}$. Here blue and red atoms denote titanium and oxygen respectively. 
S3. Average Ti-O bond length in $a-\mathrm{TiO}_{2}$ and their distortion index
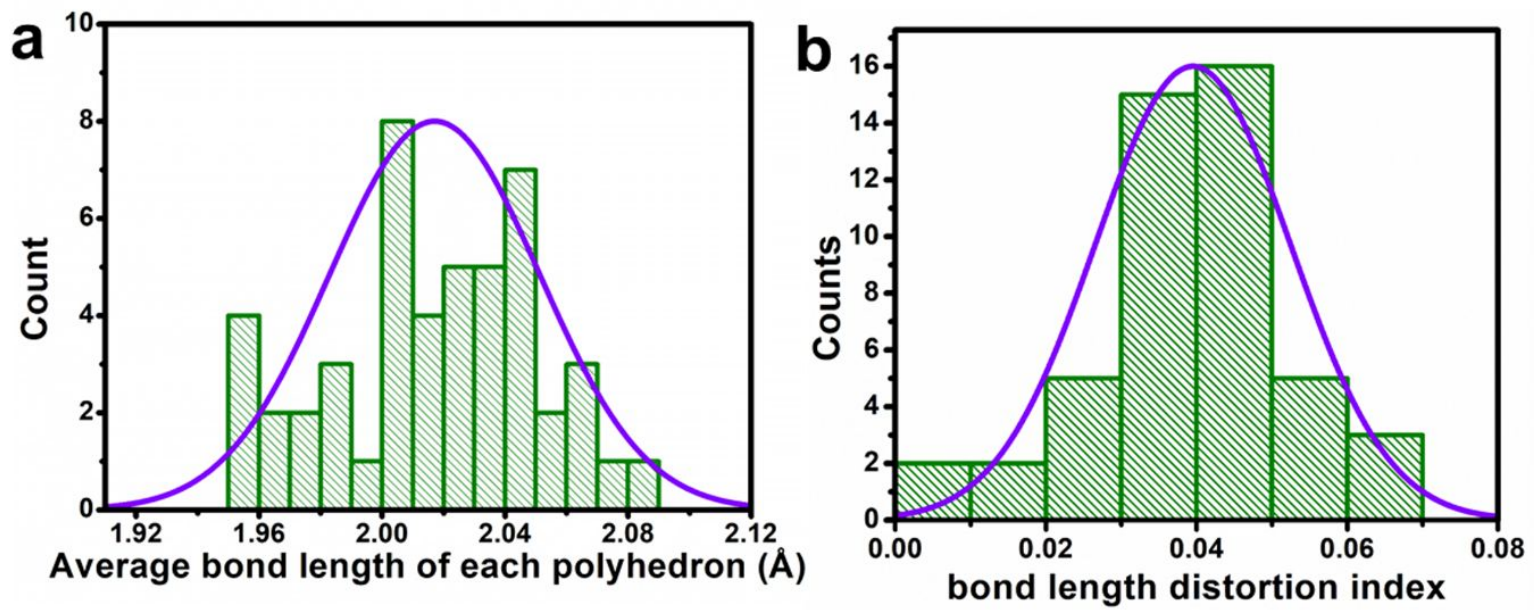

Figure S3. (a) Average Ti-O bond length of 48 polyhedra in DFT simulated $a-\mathrm{TiO}_{2}$ structure and (b) distortion in their bond length.

Here, distortion index $(D I)$ in bond length is defined as, ${ }^{4-5}$

$$
D I=\frac{1}{n} \sum_{i=1}^{n} \frac{\left|b_{i}-b_{a v}\right|}{b_{a v}}
$$

where, $b_{a v}$ is the average bond length of each polyhedron and $b_{i}$ is the distance of the $i$-th oxygen atom from the central $\mathrm{Ti}$ atom. 
S4. Density of states of oxygen vacancies at different charge state
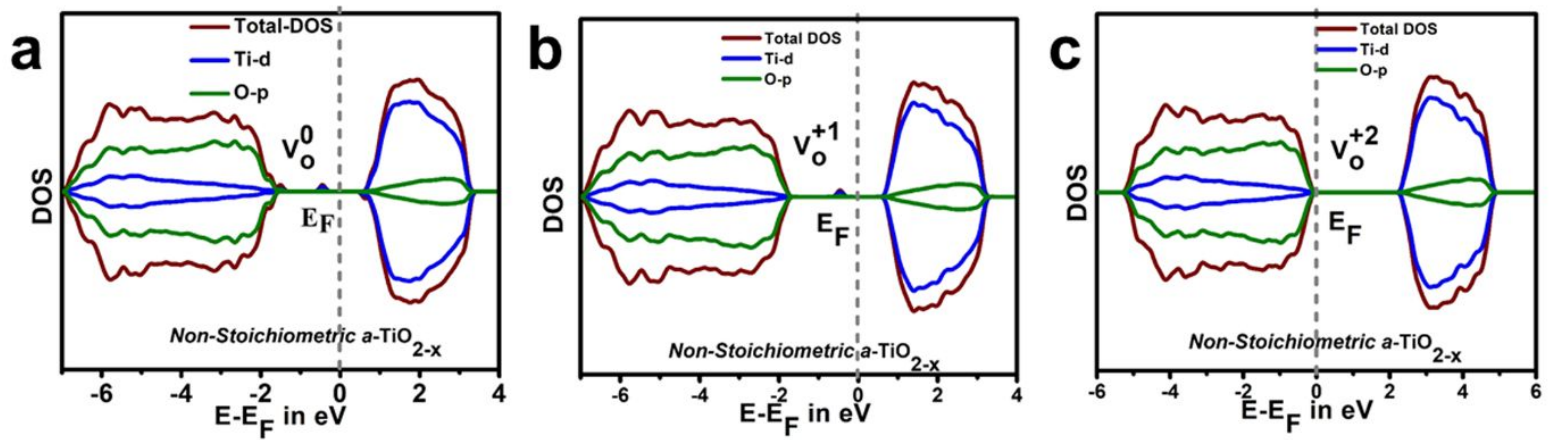

Figure S4. Projected density of states of different oxygen vacancies (a) $V_{O}^{0}$, (b) $V_{O}^{+1}$ and (c) $V_{O}^{+2}$ Here blue and brown coloured lines describe the contribution from Ti- $d$ and O- $p$ orbitals respectively. 


\section{S5. The details of the Monte-Carlo (MC) Simulations}

We briefly describe below the Metropolis-Hastings algorithm based Monte Carlo simulation scheme, ${ }^{6-8}$ which has been employed in the present study to observe the formation of the conducting filaments. ${ }^{9-11}$

\section{a. The balance condition and acceptance probability}

Suppose, we move from step $i$ to $i+1$ in the Markov chain then change in microstate probability $\wp_{m}$ can be written as

$$
\wp_{m}(i+1)=\wp_{m}(i)-\sum_{n} T_{m n} \wp_{m}(i)+\sum_{n} T_{n m} \wp_{n}(i)
$$

Here $T_{m n}$ denotes the transition probability from state $m$ to $n$, similarly $T_{n m}$ for state $n$ to $m$. Since $\wp_{n}(i+1)=\wp_{n}(i)$ is always true for the Markov Process and also at equilibrium probability do not change with time, therefore

$$
T_{m n} \wp_{m}(i)=T_{n m} \wp_{n}(i) \text { for all } m, n
$$

This is detailed balanced equation using it we can write the transition probability as the product of two terms.

$$
T_{m n}=\alpha_{m n} P_{m n}^{a c c}
$$

$\alpha_{m n}$ is the random move proposal probability from state $m$ to $n$ and $P_{m n}^{a c c}$ is the acceptance probability, now the using the detailed balance condition (3) we get:

$$
\frac{P_{m n}^{a c c}}{P_{n m}^{a c c}}=\frac{\alpha_{n m} \wp_{n}}{\alpha_{m n} \wp_{m}}
$$

For symmetric moves, the move proposal probabilities are equal in forward and reverse directions $\alpha_{m n}=\alpha_{n m}$, therefore,

$$
\frac{P_{m n}^{a c c}}{P_{n m}^{a c c}}=\frac{\wp_{n}}{\wp_{m}}
$$

We have $\wp_{m}=e^{-\beta U_{m}} / Z$ and $\wp_{n}=e^{-\beta U_{n}} / Z$ therefore we get 


$$
\frac{P_{m n}^{a c c}}{P_{n m}^{a c c}}=\frac{e^{-\beta U_{n}} d r^{N} / Z}{e^{-\beta U_{m}} d r^{N} / Z}=e^{-\beta\left(U_{n}-U_{m}\right)}
$$

This is the acceptance criterion of the MC moves; we can choose the move as if it satisfy the above equation. In the Metropolis algorithm the acceptance criterion is given by

$$
P_{m n}^{a c c}=\min \left[1, e^{-\beta\left(U_{n}-U_{m}\right)}\right]
$$

If, $U_{n}<U_{m}$ then $P_{m n}^{a c c}=1$ else, $P_{m n}^{a c c}=\frac{1}{e^{\beta\left(U_{n}-U_{m}\right)}}$

\section{b. The details of the Metropolis algorithm is given as follows}

1) Consider a canonical ensemble with $N$ number of particles, which are randomly distributed in a cubical box of length $L$.

2) Determine an energy function $\left(U_{m}\right)$ to describe the ensemble.

3) Pick up a particle $i$ randomly from the distribution.

4) Apply $\Delta r$ amount of perturbation on each of its $x, y$, and $z$ coordinates of $i$-th particle.

5) Calculate the new energy function ( $U_{n}$ ) to find the change in energy $\Delta U=U_{m}-U_{n}$ caused due to the perturbation.

6) Use Metropolis algorithm to decide the acceptance of movement, if $\Delta U<0$, accept the change and update the configuration, else in case of $\Delta U<0$, the configuration is accepted with a certain probability, the probability function defined as $p_{\alpha}=e^{-\Delta U / k \mathrm{~T}}$. Generate a random number, $\alpha \in[0,1]$, if $\alpha<p_{\alpha}$ then the new configuration is accepted otherwise reject the move and update the configuration accordingly.

7) Repeat the process from step-3 onwards for all other particles to achieve the final configuration. 
c. The optimised parameters used in MC simulation

\begin{tabular}{|c|c|}
\hline Simulation box size (L) & $50 \mathrm{~nm}$ \\
\hline A & $0.753 \times 10^{-3} \mathrm{eV} / \AA$ \\
\hline B & $11.83 \mathrm{eV} / \AA^{2}$ \\
\hline C & $383.45 \mathrm{eV} / \AA^{6}$ \\
\hline$U_{0}$ & $10^{8} \mathrm{eV} / \mathrm{cm}$ \\
\hline$\theta$ & $12^{\circ}$ \\
\hline Number of particles $(n)$ & 108 \\
\hline
\end{tabular}




\section{References}

(1) Sinha, A.; Sagdeo, A.; Gupta, P.; Upadhyay, A.; Kumar, A.; Singh, M.; Gupta, R.; Kane,

S.; Verma, A.; Deb, S. Angle Dispersive X-ray Diffraction Beamline on Indus-2 Synchrotron Radiation Source: Commissioning and First Results, J. Phys: Conf. Ser. IOP Publishing: 2013, $425,072017$.

(2) Sinha, A.; Singh, M.; Achary, S.; Sagdeo, A.; Shukla, D.; Phase, D. Crystal Field Splitting and Spin States of Co Ions in Cobalt Ferrite with Composition $\mathrm{Co}_{1.5} \mathrm{Fe}_{1.5} \mathrm{O}_{4}$ using Magnetization and X-ray Absorption Spectroscopy Measurements. J. Magn. Magn. Mater. 2017, 435, 87-95.

(3) Ravel, B.; Newville, M. ATHENA, ARTEMIS, HEPHAESTUS: Data Analysis for X-ray Absorption Spectroscopy using IFEFFIT. J. Synchrotron Radiat. 2005, 12 (4), 537-541.

(4) Momma, K.; Izumi, F. VESTA: A Three-Dimensional Visualization System for Electronic and Structural Analysis. J. Appl. Crystallogr. 2008, 41 (3), 653-658.

(5) Momma, K.; Izumi, F. VESTA 3 for Three-Dimensional Visualization of Crystal, Volumetric and Morphology Data. J. Appl. Crystallogr. 2011, 44 (6), 1272-1276.

(6) Chib, S.; Greenberg, E. Understanding the Metropolis-Hastings Algorithm. Am. Stat. 1995, 49 (4), 327-335.

(7) Roberts, G. O.; Rosenthal, J. S. Optimal Scaling for Various Metropolis-Hastings Algorithms. Statist. Sci. 2001, 16 (4), 351-367.

(8) Gagniuc, P. A. Markov Chains: from Theory to Implementation and Experimentation; John Wiley \& Sons, New Jersey: 2017.

(9) Tian, H.; Wang, X.-F.; Mohammad, M. A.; Gou, G.-Y.; Wu, F.; Yang, Y.; Ren, T.-L. A Hardware Markov Chain Algorithm Realized in a Single Device for Machine Learning. Nat. Commun. 2018, 9 (1), 4305. 
(10) Aldana, S.; Roldán, J.; García-Fernández, P.; Suñe, J.; Romero-Zaliz, R.; JiménezMolinos, F.; Long, S.; Gómez-Campos, F.; Liu, M. An In-depth Description of Bipolar Resistive Switching in $\mathrm{Cu} / \mathrm{HfO}_{\mathrm{x}} / \mathrm{Pt}$ Devices, a 3D Kinetic Monte Carlo Simulation approach. J. Appl. Phys. 2018, 123 (15), 154501.

(11) Pan, F.; Subramanian, V. In Kinetic Monte Carlo Simulation of Resistive Switching and Filament Growth in Electrochemical RRAMS, 68th Device Research Conference, IEEE: 2010; pp 255-256. 